\title{
Me Without My Smartphone? Never! Predictors of Willingness for Smartphone Separation and Nomophobia
}

\author{
Ricardo Muench $^{(\bowtie)}$ and Catharina Muench \\ Julius-Maximilians University of Wuerzburg, \\ Oswald-Kuelpe-Weg 82, 97074 Würzburg, Germany \\ ricardo.muench@uni-wuerzburg.de
}

\begin{abstract}
The smartphone as a ubiquitous mobile computer can be regarded as a 'digital companion', being with us $24 / 7$ and supporting us in every aspect of modern life. This companionship might lead to a psychological attachment to the device comparable to the attachment to social (human) partners, and ultimately even compulsive usage. Here, a relevant construct is the fear of missing out (short: FoMO) leading to a constant checking of one's phone. Furthermore, users can develop a fear called 'nomophobia' (no-mobile-phone phobia). Despite this fear, resolutions to reduce overuse also known as a 'digital detox' is an emerging trend. Our research aims at revealing predictors of nomophobia and the willingness to limit one's smartphone usage, and which sub-factors of nomophobia are dominating. $\mathrm{N}=220$ participants filled out an online survey including factors like compulsive usage, FoMO, nomophobia, frequency of smartphone usage and willingness to separate from one's smartphone. Multiple regression analyses revealed potential predictors for nomophobia and willingness for smartphone separation.
\end{abstract}

Keywords: Smartphone separation $\cdot$ Nomophobia $\cdot$ Digital companion

\section{Introduction}

There are numerous occasions in everyday life, in which people are seen using their smartphone non-stop. It is used in every situation thinkable - on public transport or at home, as a primary activity or as a second screen when watching television. While its basic function is a means to communicate, it can also be used for work-related tasks, to access information or to entertain oneself. The constitution of the smartphone as an ubiquitous and multi-functional mobile computer is also reflected in daily usage time, which was an average of $3 \mathrm{~h}$ and $35 \mathrm{~min}$ in the US in 2018 [16]. There are even fashion trends like the smartphone neck-strap, which always enable the (in this case predominantly female) users to have their device close-by. It allows direct smartphone access even without pulling it out of pants pockets or bags. It seems that the time spent with this technology needs to be maximized at all cost.

There is a lot of research regarding mobile phone usage and the effects on the user. Studies address questions concerning a typical psychological predisposition of an 
'over-user' [3] or decreases in productivity due to constant distractions by the smartphone [9]. Consequently, there is also a field of research interested in the benefits of consciously limiting one's smartphone usage by conducting a digital detox [1, 2, 14]. But are people willing to separate from their own smartphone? And if so, what factors influence and predict the willingness to engage in such self-limitation?

\section{Theoretical Framework}

Due to the multi-functionality of smartphones, they can be described as the perfect devices to gratify users' needs. As described above, users stay connected with their phone throughout the day and interact with it frequently. The smartphone can be conceptualized as a "digital companion", which is more than mere technical equipment [5]. The interaction of users with their device can even evolve in human-like relationships and psychological attachment comparable to other social (human) partners [4].

The tendency to form social and emotional attachments is deeply rooted in humans, and even extends to the attachment with non-living objects, e.g. technology. Especially younger age-groups seem prone to form such relationships with the smartphone: they are constantly trying to stay connected with the device and feel distressed when separated from it [11]. This attachment even goes so far that the smartphone is used while driving a car. Using a representative sample of young drivers (17-28 years) it was determined that high mobile attachment predicts distracted driving behavior, e.g. by checking social media app notifications [17]. People highly attached to their smartphone are prone to an overuse of the device, also labeled a problematic smartphone use. This problematic overuse is often linked to negative feelings like stress and decreased life satisfaction [12].

Research has also been looking at the usage motives to explain why we are using the smartphone as frequently as we do. One motive is the constant connectedness with friends, family and all types of acquaintances by communicating directly or using social media applications. A possible underlying factor is the so called 'fear of missing out' (short: FoMO) [13]. It is defined as a omnipresent fear, that others might have exciting experiences, which we do not know about or cannot attend to. In a study about problematic smartphone overuse, FoMo was shown to be related to high social media usage [10]. FoMO is argued to lead to increased smartphone usage, as it gives users the opportunity to gather information about related people and ongoing events in one's peer group. This is further underlined by the findings of Wolniewicz and colleagues: high FoMO was shown to be related to increased social uses of the smartphone compared to functional uses [18]. Therefore, FoMO seems to be predicting not only increased smartphone usage time overall, but also social application usage especially.

In this context, users can develop a fear called 'nomophobia', an acronym for 'no mobile phone phobia'. It describes the fear of not being in touch with the smartphone and the various functions it enables. Thus, this concept contains four factors: (1) not being able to communicate, (2) losing the connectedness with the online (social) world, (3) discomfort of losing pervasive access to information and (4) giving up the convenience a smartphone gives [19]. A study with students from India showed, that approximately $75 \%$ of participants suffered from nomophobia [15]. 
Despite these fears and other psychological factors like mobile attachment, or maybe because of them, resolutions to limit smartphone use also known as 'digital detoxing' is an emerging trend. Digital detox is mostly conducted in a private social environment, in order to improve the quality time spent with family and friends. However, studies analyzing the effects of a short-term separation from one's own smartphone showed that this may lead to higher levels of anxiety [6], mediated by FoMO [14] and an increase of blood pressure and a decrease of task performance [7]. Research analyzing long-term separation effects of smartphone separation, like a digital detox, show contrary results as participants report significantly lower stress levels compared to the control group using their smartphones regularly [2].

Based on previous research, there are several factors that seem to be relevant regarding the willingness to separate from one's smartphone. Nevertheless, the most intuitive predictor is nomophobia, as the fear to be without one's smartphone should influence the willingness to engage in a limitation of said device. To further analyze which users experience nomophobia in the first place we ask for underlying factors of this fear:

RQ1: Which of the factors FoMO, mobile attachment, compulsive smartphone usage, frequency of smartphone usage and well-being significantly predict nomophobia?

Furthermore, we were interested in examining the relevant factors in willingness for smartphone separation besides nomophobia:

RQ2: Which of the previous factors plus nomophobia significantly predict the willingness for smartphone separation?

\section{Method}

\subsection{Participants}

The sample consisted of 220 (139 female, 80 male, 1 unspecified gender) people, recruited by posting in social networking sites. Participation was voluntary. The age ranged from 12 to 77 years with a mean age of 27.89 years $(S D=11.58)$. Approximately $80 \%$ of participants were of ages 30 and younger. Most participants were enrolled in education (student, apprentice, etc.), only $34.7 \%$ were employed.

\subsection{Measures}

To assess the underlying factors of nomophobia and willingness to separate from one's smartphone, participants filled out an online questionnaire, which was created with an online tool called "SosciSurvey". The questionnaire included various scales, as well as several demographic questions.

Frequency of smartphone usage was gathered by asking how long in minutes the smartphone was used on average daily $(M=287.74 \mathrm{~min}, S D=180.09 \mathrm{~min})$. 
Willingness for smartphone separation was measured with a single item stating 'I would be willing to give up my smartphone for one week'. The item could be answered on a 5-point Likert scale from $1=$ 'strongly disagree' to $5=$ strongly agree' $(M=3.14, S D=1.49)$.

Well-Being was assessed with the 'Flourishing Scale' by Diener and colleagues [8]. The scale consists of eight items, all answerable on a 7-point Likert scale from $1=$ 'strongly disagree' to $7=$ 'strongly agree' $(M=5.67, S D=.88)$.

Mobile Attachment was measured by the Mobile Attachment Scale [11], consisting of 10 Items with a 5-point Likert scale from $1=$ 'strongly disagree' to $5=$ 'strongly agree'. $(M=2.70, S D=.78)$.

For the measurement of compulsive smartphone usage, we used a scale consisting of 13 Items with a 7-point Likert scale from $1=$ 'strongly disagree' to $7=$ 'strongly agree' [12] $(M=2.80, S D=1.02)$.

To assess FoMO we used the Fear of Missing Out Scale [13]. It consists of ten items on a 5-point Likert scale from $1=$ 'strongly disagree' to $5=$ 'strongly agree' $(M=2.65, S D=.84)$.

To conclude, nomophobia was measured with the Nomophobia Questionnaire (NMP-Q) [19] with 20 Items on a 7-point Likert scale from 1 = 'strongly disagree' to 7 = 'strongly agree'. The 20 items are summed up into one single score, which consequently ranges from 20 to 140 . Based on the score, participants can be classified regarding their nomophobia: $20=$ complete absence of nomophobia; $21-59=$ mild level of nomophobia; $60-99=$ moderate level of nomophobia; $100-140=$ severe nomophobia. In our survey, the participants reach an average score of $(M=61.86$, $S D=24.18$ ), which is a moderate level of nomophobia.

\section{Results}

To answer the two research questions, multiple linear regressions were conducted in order to analyze the prediction of nomophobia and the willingness for smartphone separation.

In RQ1, we meant to examine if and to what extent the factors FoMO, mobile attachment, compulsive smartphone usage, frequency of smartphone usage and wellbeing significantly predict nomophobia. A significant regression equation was found ( $F$ $(5,214)=111.79, p<.001)$, with an $R^{2}$ of .85 (adjusted $R^{2}=.72$ ). Participants' predicted nomophobia is equal to $.46+.11$ (compulsive smartphone usage) -.08 (well-being) +.26 (FoMO) +.57 (mobile attachment) .04 (frequency of smartphone usage). Well-being $(p=.04)$, FoMO $(p<.001)$, and mobile attachment $(p<001)$ were significant predictors. Compulsive usage was a near-significant predictor $(p=.06)$.

RQ2 asked for the predictors of willingness for smartphone separation. A significant regression equation was found $(F(6,213)=12.63, p<.001)$, with an $R^{2}$ of .51 (adjusted $R^{2}=.26$ ). Participants' predicted willingness for smartphone separation is 
equal to $3.85+.10$ (compulsive smartphone usage) +.11 (well-being) +.12 (FoMO) -.19 (mobile attachment) -.07 (frequency of smartphone usage) -.44 (nomophobia). Only nomophobia was a significant predictor $(p<.001)$. Well-being $(p=.06)$ and mobile attachment $(p=.10)$ were near-significant predictors.

As nomophobia was the only significant predictor for the willingness for smartphone separation, we conducted an additional exploratory regression analysis including the four sub-factors of nomophobia: not being able to communicate, losing connectedness, not being able to access information and giving up convenience. We wanted to examine which sub-factor significantly predicts the willingness for smartphone separation. A significant regression equation was found $(F(4,215)=16.46, p<.001)$, with an $R^{2}$ of .48 (adjusted $R^{2}=.23$ ). Participants' predicted willingness for smartphone separation was equal to $4.95-.24$ (not being able to communicate) -.14 (losing connectedness) -.09 (not being able to access information) -.10 (giving up convenience). Only 'not being able to communicate' was a significant predictor $(p=.01)$.

\section{Discussion and Outlook}

We intended to analyze the underlying factors of nomophobia and the willingness to separate from one's smartphone. Regarding nomophobia, there were several significant predictors. Higher levels of FoMO and mobile attachment lead to higher levels of nomophobia. From a theoretical standpoint, both FoMO and mobile attachment are closely linked to nomophobia. The fear of missing out on social events can be a relevant factor in developing a fear to be without one's smartphone. Additionally, high intensity of the attachment formed with the technological device predicts nomophobia, as users do not want to lose their digital companion. Nomophobia was also negatively predicted by well-being. It seems like smartphone usage does not only lead to negative emotions in users [12], but an overall good mood decreases the fear of not being in touch with the smartphone. Compulsive usage and frequency of usage were not statistically significant.

Research question 2 focused on the underlying factors of the willingness to separate from one's smartphone. Our analysis showed, that only nomophobia was a significant predictor. Whereas this relationship is rather obvious, it is interesting to note that no other predictors could be found. To gain further insights on the role of nomophobia, we looked at the four sub-factors that nomophobia consists of. Only the first sub-factor 'not being able to communicate' was a significant predictor in this analysis, which was negatively associated. This result showed, that it is the most basic function of the smartphone, namely the enablement of communication with friends and family, which predicts the (un-)willingness to separate from one's smartphone. This relationship might be even of increased importance in times of a global health crisis like we are experiencing now with the new-found Covid-19 virus. Direct contact is restricted in numerous countries; therefore, many people rely on communication devices to stay in touch with their social surroundings.

Some experimental limitations can be discussed. We did not assess the entirety of possible factors, as a voluntary participation in an online survey should not be too extensive. Due to this, we had to exclude possible factors like stress induced by the 
phone. We argue for further research in this field of study, to broaden the knowledge which factors predict the willingness to participate in a digital detox. It would be interesting to examine the typical predispositions of users limiting their smartphone usage, e.g. by looking at personality factors. Experiments like the present study help us understand the close connectedness users establish with their smartphone as a digital companion.

\section{References}

1. Adelhardt, Z., Markus, S., Eberle, T.: Teenagers' reaction on the long-lasting separation from smartphones, anxiety and fear of missing out. In: Proceedings of the 9th International Conference on Social Media and Society, pp. 212-216 (2018)

2. Anrijs, S., et al.: MobileDNA: relating physiological stress measurements to smartphone usage to assess the effect of a digital detox. In: Stephanidis, C. (ed.) HCI 2018. CCIS, vol. 851, pp. 356-363. Springer, Cham (2018). https://doi.org/10.1007/978-3-319-92279-9_48

3. Bianchi, A., Phillips, J.G.: Psychological predictors of problem mobile phone use. CyberPsychol. Behav. 8(1), 39-51 (2005)

4. Bodford, J.E., Kwan, V.S., Sobota, D.S.: Fatal attractions: attachment to smartphones predicts anthropomorphic beliefs and dangerous behaviors. Cyberpsychol. Behav. Soc. Netw. 20(5), 320-326 (2017)

5. Carolus, A., Binder, J.F., Muench, R., Schmidt, C., Schneider, F., Buglass, S.L.: Smartphones as digital companions: characterizing the relationship between users and their phones. New Media Soc. 21(4), 914-938 (2019)

6. Cheever, N.A., Rosen, L.D., Carrier, L.M., Chavez, A.: Out of sight is not out of mind: the impact of restricting wireless mobile device use on anxiety levels among low, moderate and high users. Comput. Hum. Behav. 37, 290-297 (2014)

7. Clayton, R.B., Leshner, G., Almond, A.: The extended iSelf: the impact of iPhone separation on cognition, emotion, and physiology. J. Comput.-Mediat. Commun. 20(2), 119-135 (2015)

8. Diener, E., et al.: New well-being measures: short scales to assess flourishing and positive and negative feelings. Soc. Indic. Res. 97(2), 143-156 (2010)

9. Duke, É., Montag, C.: Smartphone addiction, daily interruptions and self-reported productivity. Addict. Behav. Rep. 6, 90-95 (2017)

10. Elhai, J.D., Levine, J.C., Dvorak, R.D., Hall, B.J.: Fear of missing out, need for touch, anxiety and depression are related to problematic smartphone use. Comput. Hum. Behav. 63, 509-516 (2016)

11. Konok, V., Pogány, Á., Miklósi, Á.: Mobile attachment: separation from the mobile phone induces physiological and behavioural stress and attentional bias to separation-related stimuli. Comput. Hum. Behav. 71, 228-239 (2017)

12. Lee, Y.K., Chang, C.T., Lin, Y., Cheng, Z.H.: The dark side of smartphone usage: psychological traits, compulsive behavior and technostress. Comput. Hum. Behav. 31, 373383 (2014)

13. Przybylski, A.K., Murayama, K., DeHaan, C.R., Gladwell, V.: Motivational, emotional, and behavioral correlates of fear of missing out. Comput. Hum. Behav. 29(4), 1841-1848 (2013)

14. Schmidt, C., Muench, R., Schneider, F., Breitenbach, S., Carolus, A.: Generation "always on" turned off. Effects of smartphone separation on anxiety mediated by the fear of missing out. In: Stephanidis, C. (ed.) HCI 2018. CCIS, vol. 851, pp. 436-443. Springer, Cham (2018). https://doi.org/10.1007/978-3-319-92279-9_58 
15. Sharma, N., Sharma, P., Sharma, N., Wavare, R.R.: Rising concern of nomophobia amongst Indian medical students. Int. J. Res. Med. Sci. 3(3), 705-707 (2015)

16. Statista. https://www.statista.com/statistics/1045353/mobile-device-daily-usage-time-in-theus/. Accessed 27 Mar 2020

17. Weller, J.A., Shackleford, C., Dieckmann, N., Slovic, P.: Possession attachment predicts cell phone use while driving. Health Psychol. 32(4), 379 (2013)

18. Wolniewicz, C.A., Tiamiyu, M.F., Weeks, J.W., Elhai, J.D.: Problematic smartphone use and relations with negative affect, fear of missing out, and fear of negative and positive evaluation. Psychiatry Res. 262, 618-623 (2018)

19. Yildirim, C., Correia, A.P.: Exploring the dimensions of nomophobia: development and validation of a self-reported questionnaire. Comput. Hum. Behav. 49, 130-137 (2015) 